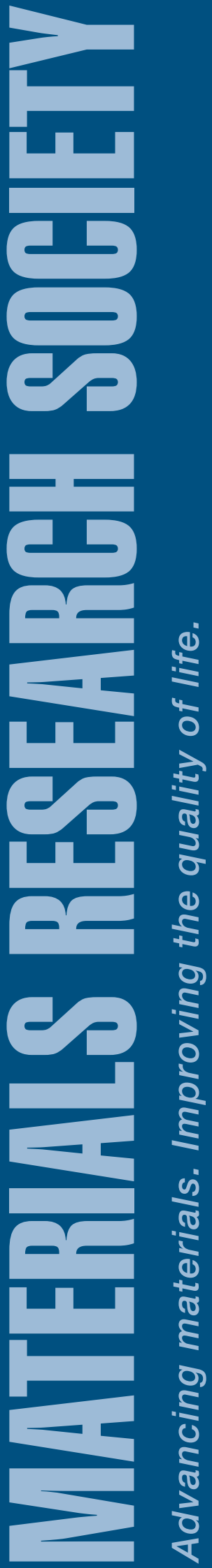

Our goal as a Society is to embrace, cultivate, and capitalize on the diversity of our members, and use this diversity as a resource for a dynamic and vibrant future.

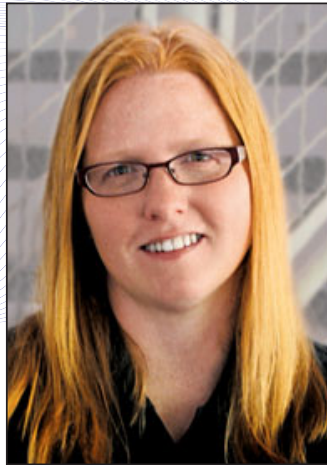

Kristi S. Anseth

2016 MRS President

\title{
Engaging and equipping members to be the future of the Society
}

"If your vision is for a year, plant wheat. If your vision is for ten years, plant trees. If your vision is for a lifetime, plant people."

I quoted that Chinese proverb in 2014 as a closing thought in my Candidate's Statement for MRS President. It spoke to my vision of serving "to plant and nurture people that would be the future of MRS." Two years later, and halfway through my presidency, those words still guide my efforts to make a difference in the future of the Society and its members. The challenge, as I see it, is to not only serve MRS members, but to engage them in activities that match their passion.

MRS has a long history of achievement based on volunteer vision and commitment. A quote by Harry Gatos, the first MRS President, was recited on the occasion of the Society's 40th anniversary: "The founding and operation of MRS was the culmination of my 10 years of frustrated effort in searching for a professional home (old, renovated, or new) for the young, homeless materials science." The MRS Spring and Fall Meetings are world-renowned for their strong scientific programming and technical content, and that is in large part due to their unique model. Society leadership literally hands over control of each meeting to five new volunteer Meeting Chairs - materials researchers working in the field with firsthand knowledge of what is most important to the community. And year after year, meeting after meeting, those Chairs, together with their Symposium Organizers and numerous other volunteers and staff, create exciting and informative events that carry on the "great meetings society" legacy.

Since its inception in late 2012, the Materials Research Society Foundation has also encouraged and funded member-proposed outreach projects such as SciBridge, ${ }^{*}$ a venture designed to connect US and African materials scientists and university students via collaborative experiments and web seminars. It also funds free and discounted electronic memberships for those studying and working in developing countries, and provides support for collaborative educational efforts such as Impact of Materials on Society (IMOS), a new undergraduate and community college course that uses multiple perspectives - engineering, anthropology, history, English, sociology, and philosophy - to explore and understand materials innovation. This is a small sampling of the valuable and inspiring accomplishments MRS has attributed to its volunteer leadership over the past 43 years. But I know we can do more.

${ }^{*}$ www.scibridge.org 
The next step for MRS is to seek Inclusive Excellence in our Society. Our membership is almost 16,000 strong. It includes men and women from more than 90 countries around the world - from the richest of nations to developing countries. Our members come from industry, academia, and national labs, and their work touches on many fields, including chemistry, biology, physics, and engineering. They have skills and expertise that range from technical and organizational, to advocacy and education. And they bring rich backgrounds, experiences, perspectives, talents, gifts, and cultures to our community. Our goal, as a Society, must be to embrace, cultivate, and capitalize on that diversity and use it as a resource for a dynamic and vibrant future. To that end, we need to ensure multiple channels are in place for input from our members, learn how to better partner with them in building new initiatives and programs, and provide avenues for efficient implementation of the best ideas. This is especially important as our Society grows in number and continues to diversify geographically and scientifically. And I encourage all of you to become part of the process.

If you are looking for a way to expand your professional network, enhance your leadership skills, or shape the future of our Society, then consider becoming a MRS volunteer. I realize that many of you are highly successful, have numerous commitments, and are balancing your personal and professional life. I am a wife, mother, scientist, editor, teacher, writer, and mentor, so I understand and value your time. However, we have volunteer opportunities that are long term and short term, which require varying skills and interests, and may very well match your passion.

Please feel free to contact Michele Feder (feder@mrs.org), Manager of Volunteer Affairs, to learn more about our volunteer opportunities. And then join us, as we continue to develop programs and plant and nurture the next generation of MRS!

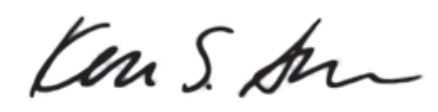

Kristi S. Anseth 2016 MRS President 